\title{
Multidimensional assessment of cervical spondylotic myelopathy patients. Usefulness of a comprehensive score system
}

\author{
Fabio Pilato ${ }^{1}$ (D) $\cdot$ Rosalinda Calandrelli ${ }^{2} \cdot$ Marisa Distefano $^{3} \cdot$ Francesco Ciro Tamburrelli $^{4,5}$
}

Received: 18 January 2020 / Accepted: 28 August 2020 / Published online: 3 September 2020

(C) The Author(s) 2020

\begin{abstract}
Objective Cervical spondylotic myelopathy (CSM) is caused by cervical spine degeneration and surgery may be beneficial, but selection for surgery might be challenging. We performed a multimodal analysis to assess predicting factors that may be useful to help surgeons in this choice.

Patients and methods We retrospectively evaluated clinical, motor evoked potentials (MEP), and MRI data of patients who undergone surgery for CSM. Seventy-six consecutive patients (46 males) were enrolled. The median age was 65.5 [IQR: 57-71] years, and the duration of symptoms was 11 [8-13] months. A multivariate analysis in order to assess predictors of outcome and ROC curve analysis were performed.

Results Thirty patients (M:18, 39.5\%) gained 6 or more points on mJOA and they were collected in good recovery group, whereas 46 patients $(60.5 \%, \mathrm{M}: 28)$ showed a fair recovery. We developed a comprehensive score system (CSS) taking into account clinical, neurophysiological, and neuroradiological data. ROC curve analysis was performed to determine the discriminative power of four models derived from the multivariate logistic regression analysis for predictors of good outcome considering only clinical variables, MRI variables, and MEP variables or considering the comprehensive model, demonstrating a good accuracy of CSS model to predict outcome.

Conclusion This study demonstrates that CSS model taking into consideration functional assessment by mJOA score, neurologic evaluation, cervical MRI, and MEP may be a feasible method to predict outcome in patients candidate to surgery, supporting surgeon's decisions both for those patients candidate to surgery and for patients in whom a "wait and see" approach could be proposed.
\end{abstract}

Keywords Magnetic resonance imaging $\cdot$ Cervical spondylotic myelopathy $\cdot$ Prognosis $\cdot$ Motor-evoked potentials $\cdot$ Myelopathy $\cdot$ Personalized medicine

Electronic supplementary material The online version of this article (https://doi.org/10.1007/s10072-020-04691-0) contains supplementary material, which is available to authorized users.

Fabio Pilato

fabio.pilato@policlinicogemelli.it

Rosalinda Calandrelli

rosalinda.calandrelli@policlinicogemelli.it

Marisa Distefano

marisa.distefano@hotmail.it

Francesco Ciro Tamburrelli

francescociro.tamburrelli@policlinicogemelli.it

1 UOC Neurologia, Dipartimento di scienze dell'invecchiamento, neurologiche, ortopediche e della testa-collo, Fondazione Policlinico Universitario A. Gemelli - IRCCS, 00168 Rome, Italy
2 UOC Radiologia e Neuroradiologia, Dipartimento di diagnostica per immagini, radioterapia oncologica ed ematologia, Fondazione Policlinico Universitario A. Gemelli - IRCCS, Rome, Italy

3 UOC Neurologia e UTN, Ospedale Belcolle, Strada Sammartinese, 01100 Viterbo, Italy

4 UOC Chirurgia Vertebrale, Dipartimento di scienze dell'invecchiamento, neurologiche, ortopediche e della testa-collo, Fondazione Policlinico Universitario A. Gemelli - IRCCS, Rome, Italy

5 Istituto di Ortopedia, Università Cattolica del Sacro Cuore, Rome, Italy 


\section{Introduction}

Cervical spondylotic myelopathy (CSM) is a neurological disorder caused by the degeneration of the spine and resultant spinal cord compression [2]. It is an ongoing process and patients present at various clinical stages of severity, ranging from no clinical sign to severe neurological impairment $[6$, 24]. It has become a prevalent cause of spinal cord dysfunction in aging population worldwide leading to severe physical disability. Due to the benign nature and insidious development of CSM, it had been less recognized until advanced medical technologies such as magnetic resonance imaging (MRI) and electrophysiological examinations became prevalent in recent decades. Surgery is a widely accepted treatment for severe CSM with neurological complications. However, for asymptomatic or mildly symptomatic patients with CSM, the optimal management remains debated and surgery is sometimes controversial [4].

MRI is a gold standard for CSM because it shows not only the anatomy and location of compression in the cervical spine but also intramedullary signal intensity changes in the cervical spinal cord suggestive of spinal cord lesion [23]. On the other hand, MRI reveals only morphology and not functionality of the spinal cord and MRI-driven decisions about the timing for surgery are controversial [20]. Neurophysiological techniques such as somatosensory-evoked potentials (SEP) [18] and motor-evoked potentials (MEP) may be useful tools to evaluate functionality of the cervical spinal cord $[3,8,20]$, and a prognostic role in CSM patients referred to surgery was reported $[3,19,20]$.

Previous studies evaluated whether clinical assessment, MRI, or MEP independently could have predicting value on surgical outcomes $[3,17,20]$, but conclusive results are still lacking even if correlations between presurgical MRI findings, neurophysiological assessment, and surgical outcomes were found $[16,20]$.

We evaluated clinical, neurophysiological, and neuroradiological data, and results linked with better outcome were used to develop a comprehensive model.

\section{Patients and methods}

We retrospectively investigated medical records, MEP, and MRI scans of patients who underwent surgery for CSM between January 2004 and December 2014 referred to our university hospital.

Diagnoses of CSM were made on the basis of the results of neurological examinations, neurophysiological tests, and diagnostic imaging using various techniques including MRI. The exclusion criteria were history of any spine surgery; thoracic or lumbar spinal diseases based on relevant clinical symptoms; and imaging tests and history of rheumatoid arthritis, cerebral palsy, or tumors.

After database revision, 76 consecutive patients ( 46 males and 30 females) were enrolled. All patients enrolled in this study had a minimum 1-year follow-up time, a complete neurological and neurophysiological assessment and underwent preoperative cervical MRI.

In all patients, the cervical cord was compressed at some point between the $\mathrm{C} 3 / 4$ and the $\mathrm{C} 6 / 7$ levels.

Surgical approaches and operated levels varied by case and were chosen based on both the patient's conditions and the surgeon's clinical experience.

Clinical assessment and 18-point modified Japanese Orthopedic Association (mJOA) scores were recorded [27]. Neurophysiological evaluation with MEP was performed in preoperative clinical evaluation period, and postoperative outcomes (6-12 months) were assessed by on-site follow-up visits.

Seventy-six patients with MRI documented CSM (50), cervical posterior longitudinal ligament ossification (4), and disc disease (22) underwent instrumented laminectomy (56), anterior cervical discectomy and fusion (14), or open door laminoplasty (6).

The median age was 65.5 [57-71] years, and the median duration of symptoms was 11 [8-13] months.

According to postsurgical mJOA score, patients were divided in 2 groups: good recovery and fair recovery groups. As previously measured, good recovery was defined as a complete recovery or improvements of at least $30 \%$ (six or more points) in follow-up mJOA evaluation compared with presurgical evaluation [3], whereas patients with suboptimal recovery were defined as less than $30 \%$ (five or lower points) in the comparative assessment. No patient showed worse scores in follow-up evaluation compared with presurgical evaluation, and no surgical complication was recorded.

\section{Clinical evaluation}

All patients underwent to a complete neurological examination, functional assessment, and neurophysiological evaluation both before and after surgery.

The clinical severity of neurologic function before and after surgery was assessed by the mJOA scoring system [27], and pyramidal signs such as Babinski's sign were recorded. Thirty patients (18 males and 12 females) showed a good recovery in follow-up visit, and 46 patients (28 males and 18 females) showed a fair recovery.

\section{Magnetic resonance imaging}

All patients underwent cervical MRI according to a standard protocol on a 1.5 T Signa Unit (General Electric Healthcare; Milwaukee, WI, USA) with a standard coil. MRI consisted of 
T1-weighted and T2-weighted images (T2wi) in the axial and sagittal planes. Being the aim of the study mainly focused on the relationship among clinical aspects, diagnostic imaging, and neurophysiological investigation, MRI evaluation was limited to the following aspects: presence of narrowing of the cervical spinal canal, presence of signal changes inside the cord, and proximo-distal extension of the cord compression (Fig. 1). Extension of cervical stenosis was considered the level between $\mathrm{C} 3$ and $\mathrm{C} 7$ in which CSF space was obliterated around the spinal cord and consequent compression of the spinal cord compared with spinal canal above $\mathrm{C} 3$ or below C7 level. Cervical lesion was defined as intramedullary hyperintensity on T2wi on sagittal and axial imaging [26]. A visual analysis at the compressed and reference levels, on sagittal and axial T2wi, was performed by an experienced neuroradiologist blinded about group assignment.

\section{Motor-evoked potentials}

A Magstim 200 stimulator (Magstim, Whitland, UK) was used to deliver transcranial and paravertebral stimuli through a 120-mm circular coil. MEP was recorded from the biceps, abductor digiti minimi, and tibialis anterior muscles in all patients. Cortical stimulation was performed at maximum output of the stimulator during tonic activation at about $20 \%$ of maximum voluntary contraction of the tested muscle following protocol previously described [8]. Paravertebral stimulation was performed at rest using a magnetic stimulus intensity of $60 \%$ of maximum magnetic stimulator output. Central motor conduction time (CMCT) was calculated by subtracting the peripheral conduction time from spinal cord to muscles from the latency of responses evoked by cortical stimulation, and results were considered abnormal if CMCT measurement, after superimposing the responses, a reproducible onset latency measured above reference values $[8,21]$.

\section{Statistical analysis}

Demographics and clinical characteristics were compared between subjects with good and fair recovery defined as gaining $\geq 6$ points at post-mJOA evaluation. For continuous measures, means and $\mathrm{SD}$, medians, and interquartile ranges [IQR] are presented and $p$ values calculated with a two-tailed $t$ test for Gaussian continuous variables and the Mann-Whitney $U$ or Kruskal-Wallis test for non-Gaussian continuous variables. Normality distribution was tested with Shapiro Wilk's test. For categorical measures, frequencies and percentages are presented and $p$ values calculated with a $\chi^{2}$ or a two-tailed Fisher's exact test as appropriate.

A multivariate analysis was performed using a logistic regression model with favorable outcome as dependent variable; except for age and sex, only variables with $p$ value less than 0.1 at univariate analysis were included into the multivariate models.

Receiver operator characteristic (ROC) curve analysis was performed to determine the discriminative power (area under the curve $[\mathrm{AUC}]$ ) of four models derived from the multivariate logistic regression analysis for good outcome: considering only clinical variables, neuroradiological variables, and MEP variables separately or considering the comprehensive model (CSS).

Since we excluded patients with missing essential data from our analysis, we did not impute for missing data. Statistical significance threshold was set at $p=0.05$.

Statistics were performed by means of the Statistical Package for Social Science (SPSS®) software version 25.

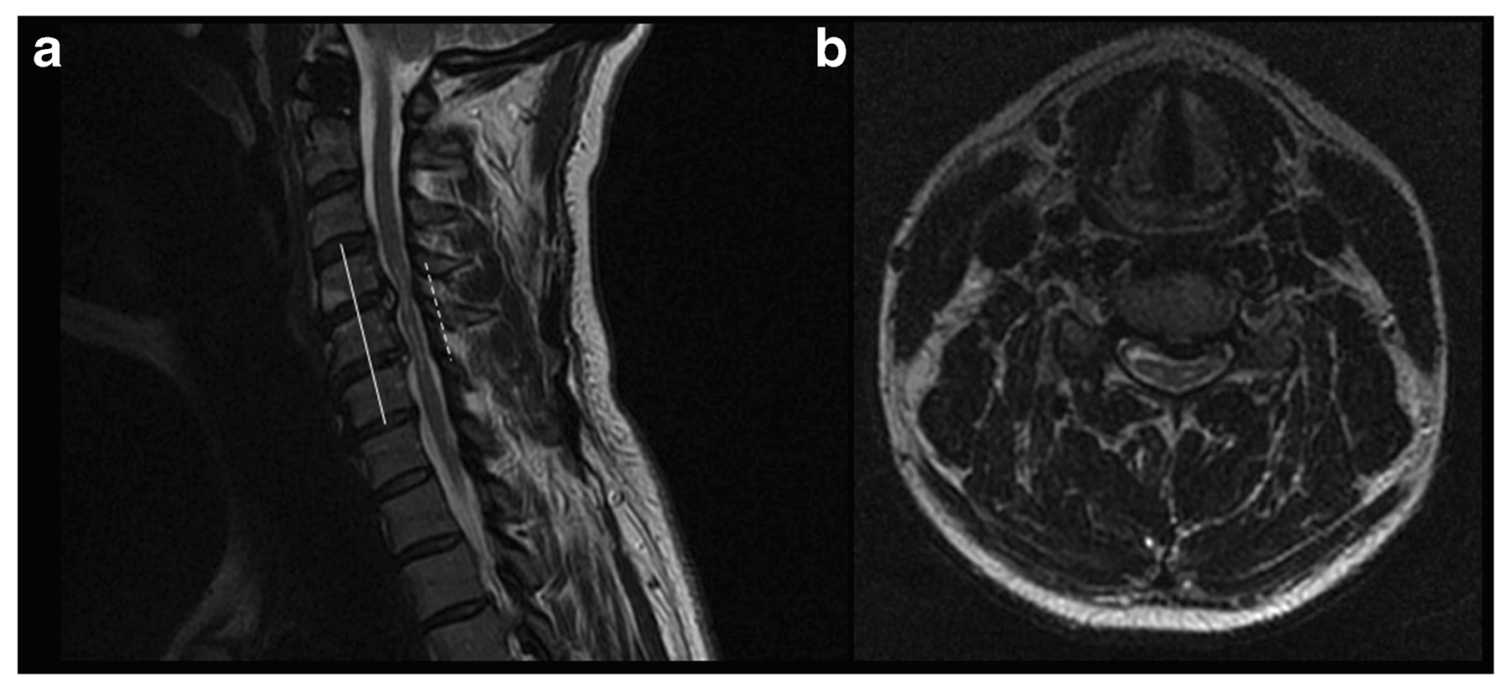

Fig. 1 MRI of a patient with central canal stenosis and myelopathy. Sagittal T2wi MR shows severe central canal stenosis extending from C4 to C6 levels with reduction of subarachnoid spaces. The central cord hyperintensity is documented to the maximum spinal cord compression level extending from C4 to C5 (a). Gray matter intramedullary T2 hyperintensity is well shown on axial T2 images (b) 


\section{Results}

Table 1 summarizes the demographics, clinical characteristics and neuroradiological and MEP results of enrolled patients.

According to postsurgical evaluation, 30 patients (M: 18, $39.5 \%$ ) gained 6 or more points on mJOA and they were collected in good recovery group, whereas 46 patients (60.5\%, M: 28) showed a fair recovery gaining 5 or less points. Presurgical assessment did not show differences in all baseline demographic characteristics between groups $(p>0.05)$, but median mJOA score was worse in good recovery group 10 [8-10] vs 11 [9-12] $(p=0.025)$, and more patients in fair group showed Babinski's sign ( $71.7 \%$ vs $43.3 \%)$ $(p=0.013)$. In enrolled patients, median duration of symptoms was 11 months [8-13] ( $p>0.05)$. Presurgical MRI evaluation showed a significant difference between groups in length of the lesion on T2wi showing longer lesions in fair recovery group $12 \mathrm{~mm}$ [12-16] compared with $6 \mathrm{~mm}$ [3-9] in good recovery group $(p<0.001)$. Presurgical MEP between groups did not show a significant difference $(p>0.05)$.
Table 2 summarizes the results of multivariate logistic regression analysis for predicting good outcome. The model included age, gender, duration of symptoms, presurgical mJOA score, Babinski's sign, baseline CMCT for biceps, abductor digiti minimi, tibialis anterior muscles, and length of the lesion on sagittal T2wi MRI. Of the other variables included, statistically significant predictors of better outcomes were shorter length of the lesion on T2wi (OR $0.69 ; 95 \%$ CI $0.55-0.81 ; p<0.001)$ and lack of Babinski's sign (OR 0.15; 95\% CI 0.03-0.79; $p=0.025$ ).

The analysis of the AUC-ROC curves for good clinical outcome shows that a model made of combining all classes of variables (clinical, neuroradiological, and neurophysiological data) obtained a AUC value of 0.92 showing the best accuracy of CSS compared with other models (Fig. 2).

\section{Discussion}

Cervical myelopathy can cause different clinical pictures varying from no symptom and sign to motor deficits and gait

Table 1 Demographics, clinical characteristics, and neuroradiological and MEP results of enrolled patients

\begin{tabular}{|c|c|c|c|c|}
\hline Demographic characteristics & All patient & Good recovery & Fair recovery & $p$ value \\
\hline Gender N. of patients (\%) & $46 \mathrm{M},(60.5 \%) 30 \mathrm{~F},(39.5 \%)$ & $\begin{array}{l}18 \mathrm{M},(60 \%) \\
12 \mathrm{~F},(40 \%)\end{array}$ & $28 \mathrm{M},(60.9 \%) 18 \mathrm{~F},(39.1 \%)$ & 0.940 \\
\hline $\begin{array}{l}\text { Age (years) } \\
\text { Median [IQR] }\end{array}$ & $65.5[57-71]$ & $67[63-68]$ & $60[54-71]$ & 0.180 \\
\hline $\begin{array}{l}\text { Duration of symptoms (months) } \\
\text { Median [IQR] }\end{array}$ & $11[8-13]$ & $8.5[7-13]$ & $12[9-13]$ & 0.068 \\
\hline $\begin{array}{l}\text { Preoperative mJOA score } \\
\text { Median [IQR] }\end{array}$ & $10[9-12]$ & $10[8-10]$ & $11[9-12]$ & 0.025 \\
\hline $\begin{array}{l}\text { Postoperative mJOA score } \\
\text { Median [IQR] } \\
\text { MRI features }\end{array}$ & $16[14-17]$ & $16[15-17]$ & $14[13-16]$ & 0.002 \\
\hline $\begin{array}{l}\text { Preoperative length of MRI lesion (mm) Median [IQR] } \\
\text { Neurological signs }\end{array}$ & $12[6-16]$ & $6[3-9]$ & $12[12-16]$ & $<0.001$ \\
\hline Babinski's sign, N. of patients, (\%) & $46(60.5 \%)$ & $13(43.3 \%)$ & $33(71.7 \%)$ & 0.013 \\
\hline $\begin{array}{l}\text { MEP Pre-surgery } \\
\text { CMCT }(\mathrm{ms}) \\
\text { Median }[\mathrm{IQR}]\end{array}$ & & & & \\
\hline $\begin{array}{l}\text { Biceps } \\
\text { (n.v. 7.6) }\end{array}$ & $6.4[5.6-6.9]$ & $6.5[5.7-7.6]$ & $6.3[5.6-6.7]$ & 0.093 \\
\hline $\begin{array}{l}\text { Abductor digiti minimi } \\
\text { (n.v. 7.7) }\end{array}$ & $11.6[9.4-13.8]$ & $12.2[10.3-14.4]$ & $10.8[8.8-13.8]$ & 0.089 \\
\hline $\begin{array}{l}\text { Tibialis anterior } \\
\text { (n.v. 17.1) } \\
\text { MEP Post-surgery }\end{array}$ & $22.6[19.9-24.8]$ & $23.4[21.2-25.5]$ & $21.6[18.2-23.9]$ & 0.079 \\
\hline $\begin{array}{l}\text { Biceps } \\
\text { (n.v. 7.6) }\end{array}$ & $6.4[5.8-6.8]$ & $6.3[5.8-6.6]$ & $6.4[5.8-6.8]$ & 0.360 \\
\hline $\begin{array}{l}\text { Abductor digiti minimi } \\
\text { (n.v. 7.7) }\end{array}$ & $11.6[9.7-13.2]$ & $11.6[9.7-13.1]$ & $11.4[9.3-14.6]$ & 0.734 \\
\hline $\begin{array}{l}\text { Tibialis anterior } \\
\text { (n.v. 17.1) }\end{array}$ & $21.4[18.9-24]$ & $21.9[19.7-24]$ & $20.8[18.8-23.2]$ & 0.372 \\
\hline
\end{tabular}

CMCT, central motor conduction time; ms, milliseconds; mm, millimeters; mJOA, modified Japanese Orthopedic Association; n.v., normal value 
Table 2 Multivariate logistic regression for predictors of good outcome

\begin{tabular}{llcr}
\hline Predictor & OR & \multicolumn{1}{l}{$95 \%$ CI } & $p$ value \\
\hline Gender (male) & 1.471 & $0.324-6.68$ & 0.617 \\
Age (younger) & 1.084 & $0.991-1.185$ & 0.077 \\
Duration of symptoms & 1.041 & $0.8-1.355$ & 0.763 \\
Presurgical mJOA score & 0.67 & $0.401-1.117$ & 0.124 \\
Babinski's sign & 0.146 & $0.027-0.788$ & 0.025 \\
CMCT (biceps) & 1.354 & $0.773-2.372$ & 0.290 \\
CMCT (abductor digiti minimi) & 1.234 & $0.896-1.701$ & 0.198 \\
CMCT (tibialis anterior) & 1.003 & $0.827-1.217$ & 0.972 \\
Length of the lesion on T2wi-MRI & 0.668 & $0.548-0.814$ & $<0.001$ \\
\hline
\end{tabular}

CMCT, central motor conduction time; mJOA, modified Japanese Orthopedic Association

disturbances associated to sphincter dysfunctions $[6,10]$. Natural history of CSM is not well characterized [1], but sometimes it may determine a progressive neurological deterioration; however, criteria for patients selection and timing for surgery are controversial [4]. Clinical history of CSM patients often requires a "wait and see period" $[5,12]$ to evaluate the progression rate of the disease, but no accordance about the best time point for surgery exists [4]. MRI or neurophysiological evaluation by MEP or SEPs may reveal early signs of cervical spinal cord involvement even if neurological evaluation might be still unremarkable $[8,14]$. On the other hand, due to time course of the CSM, at some points of the disease, some or all diagnostic exams such as MRI or neurophysiological evaluation may show signs of myelopathy $[6,24]$.

Because patients with CSM are at increased risks of neurological deterioration or spinal cord injury with nonoperative management and early surgery was linked with better outcomes, there is a trend toward attention to mildly symptomatic CSM [4].

Surgery is beneficial at a certain point of the disease in some patients $[4,15]$; however, there is not accordance about timing for surgery [9], and no test is validated to support clinician in decision-making process or in patients selection.

Usually in diagnostic workup of CSM patients, after neurological assessment, neurophysiological and neuroradiological evaluations may be suggested [14,30], and each of these examinations may give a piece of information about underlying myelopathy, and some diagnostic and treatment algorithms have been developed accordingly [22]

Neurological examination may reveal signs of impairment of central motor pathways $[10,11]$, and we found a significant negative prognostic value of Babinski's sign in the multivariate analysis (Table 2).

Neurophysiological assessment either by SEPs $[18,19]$ or MEP [3] demonstrated to be useful in evaluation of CSM patients in pre- and postsurgical period.
MEP may be a useful neurophysiological tool in diagnostic process [8] by evaluation of CMCT, muscle responses, and interside difference according to previous studies [8] and current guidelines [21]. Moreover, MEP may show an early increase of CMCT of upper limbs when signs of involvement of cervical spinal cord in these muscles are still lacking $[8,14]$. Indeed, in CSM patients, clinical signs might not be present in the initial phase of the disease when cervical spinal cord compression may cause only involvement of lower limbs with pure paraparesis [7]. Moreover, the prognostic value of MEP and SEP has also been described [3, 14]. We evaluated CMCT values of both sides for each muscle, because CMCT it is an indirect parameter of the cortico-spinal tract involvement [3, 7].

MRI has a leading role in diagnostic process giving information about level of stenosis and intramedullary lesions [17] but conflicting results raised about increase signal intensity on cervical MRI and its correlation with clinical features and outcomes $[17,26]$. We found that the length of spinal cord lesion detected on T2wi-MRI had a significant negative prognostic value in CSM patients. We performed a visual and not a morphometric evaluation of cervical MRI for a number of reasons. Several studies have already performed morphometric analyses about cervical stenosis comparing spinal canal and spinal cord diameters along with CSF signal around the cord or angles $[13,25]$. Moreover, due to the highly mobile nature of the cervical spine and the fact that usually most MRIs are obtained only in one single position, dynamic cord compression can be an elusive diagnosis that is often missed and not well-understood [29] especially when stenosis involves several levels. In fact, even if cervical canal is not stenotic on rest cervical MRI, intramedullary lesion on MRI may be discovered [29]; conversely stenotic cervical canal may be not associated with intramedullary high signal confirming a dynamic process at the basis of spondylotic myelopathy [28].

Multivariate analysis did not show a prognostic value of $\mathrm{CMCT}$ alone. On the other hand, analysis of the AUC-ROC curves for good clinical outcome shows that a model made of combining all classes of variables obtained the maximum AUC, and the accuracy of the model reaches its best value (Fig. 2). Conversely, a model made of only clinical or only neuroradiological or only neurophysiological variables alone has a small predictive power, confirming the usefulness of the CSS.

We propose a simple, easily assessable and feasible method to help clinicians in evaluating CSM patients especially those in whom surgical approach is not a straightforward decision and a "wait and see" period may be advisable. Moreover, CSS may be a useful tool to assess postsurgical outcome.

This study demonstrates that CSS taking into consideration neurologic, cervical MRI, and MEP results may be a feasible and helpful method to forecast postsurgical outcome and to guide clinical decision in CSM patients candidate to surgery. 


\section{Clinical Model}
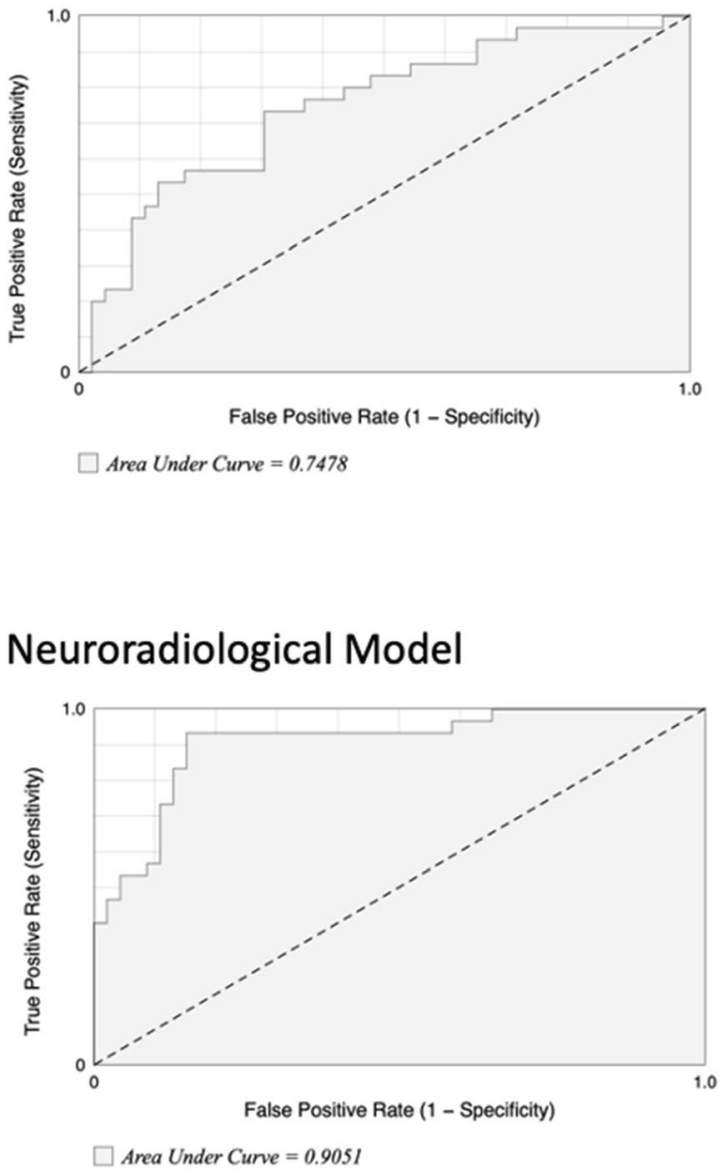

Neurophysiological Model

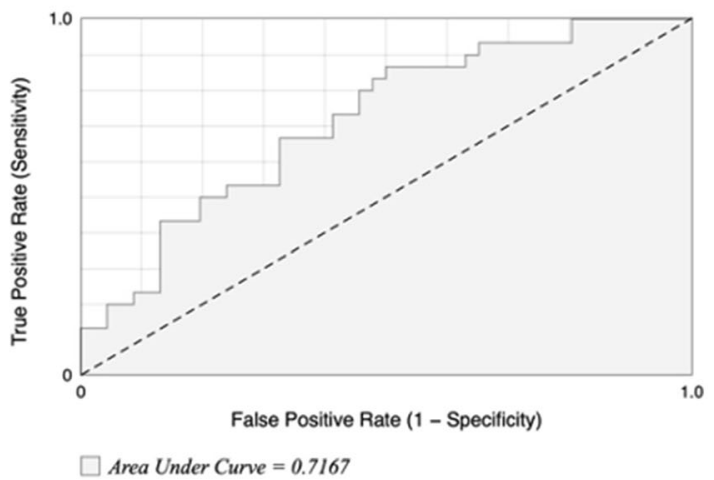

Comprehensive Score System Model

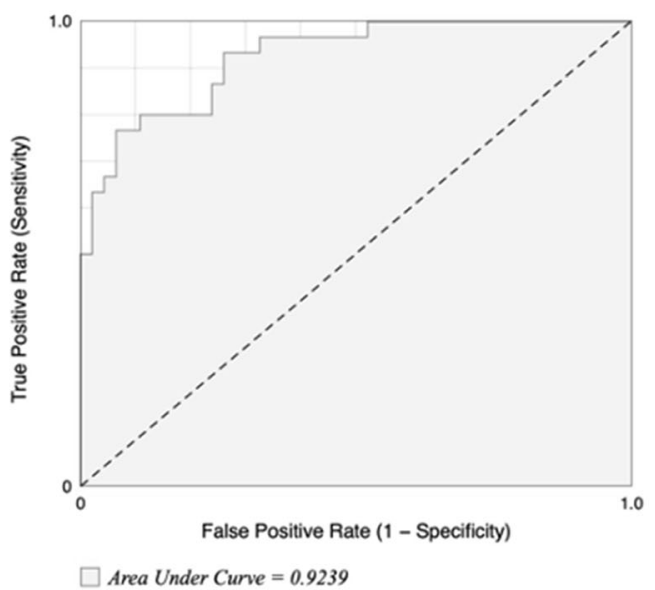

Fig. 2 ROC curve analysis of partial and comprehensive models. Figure shows ROC curve analysis of each single model and the comprehensive model. Comprehensive score system showed the best accuracy (AUC: 0.92) compared with each partial model

Further studies should be performed in subgroups of patients to assess whether, even if surgery could be less effective [15] in term of recovery, it might be beneficial in preventing progression of neurologic impairments.

\section{Limitations}

This study has some limitations: first, the retrospective design and the consequent use of post hoc hypotheses. Then, patients were allocated to surgery at the discretion of the surgeon, so selection bias cannot be excluded, and prospective studies are warranted to confirm these results in a larger population and to evaluate whether CSS may be helpful in managing decisionmaking process.

\section{Conclusion}

CSS may be a simple, practical, and useful tool to help clinicians in evaluating CSM patients in whom cervical surgery may be a therapeutic option or in whom "wait and see" period is a more advisable option.

Acknowledgements Open access funding provided by Università Cattolica del Sacro Cuore within the CRUI-CARE Agreement.

\section{Compliance with ethical standards}

Conflict of interest The authors declare that they have no conflict of interest.

Ethical approval All procedures performed in studies involving human participants were in accordance with the ethical standards of the institutional and/or national research committee and with the 1964 Helsinki declaration and its later amendments or comparable ethical standards.

Informed consent Informed consent was obtained from all individual participants included in the study.

Open Access This article is licensed under a Creative Commons Attribution 4.0 International License, which permits use, sharing, adaptation, distribution and reproduction in any medium or format, as long as you give appropriate credit to the original author(s) and the source, provide a link to the Creative Commons licence, and indicate if 
changes were made. The images or other third party material in this article are included in the article's Creative Commons licence, unless indicated otherwise in a credit line to the material. If material is not included in the article's Creative Commons licence and your intended use is not permitted by statutory regulation or exceeds the permitted use, you will need to obtain permission directly from the copyright holder. To view a copy of this licence, visit http://creativecommons.org/licenses/by/4.0/.

\section{References}

1. Baron EM, Young WF (2007) Cervical spondylotic myelopathy: a brief review of its pathophysiology, clinical course, and diagnosis. Neurosurgery 60(1 Supp1 1):S35-S41

2. Brain WR, Northfield D, Wilkinson M (1952) The neurological manifestations of cervical spondylosis. Brain J Neurol 75(2):187225

3. Capone F, Tamburelli FC, Pilato F, Profice P, Ranieri F, Di Iorio R, Iodice F, Musumeci G, Di Lazzaro V (2013) The role of motorevoked potentials in the management of cervical spondylotic myelopathy. Spine J Off J North Am Spine Soc 13(9):1077-1079

4. Chen Y-C, Kuo C-H, Cheng C-M, Wu J-C (2019) Recent advances in the management of cervical spondylotic myelopathy: bibliometric analysis and surgical perspectives. J Neurosurg Spine 31(3):299-309

5. Chikuda H, Ohtsu H, Ogata T, Sugita S, Sumitani M, Koyama Y, Matsumoto M, Toyama Y, OSCIS investigators (2013) Optimal treatment for spinal cord injury associated with cervical canal stenosis (OSCIS): a study protocol for a randomized controlled trial comparing early versus delayed surgery. Trials $14: 245$

6. Chiles BW, Leonard MA, Choudhri HF, Cooper PR (1999) Cervical spondylotic myelopathy: patterns of neurological deficit and recovery after anterior cervical decompression. Neurosurgery 44(4):762-769 discussion 769-770

7. Di Lazzaro V, Restuccia D, Colosimo C, Tonali P (1992) The contribution of magnetic stimulation of the motor cortex to the diagnosis of cervical spondylotic myelopathy. Correlation of central motor conduction to distal and proximal upper limb muscles with clinical and MRI findings. Electroencephalogr Clin Neurophysiol 85(5):311-320

8. Di Lazzaro V, Oliviero A, Profice P, Ferrara L, Saturno E, Pilato F, Tonali P (1999) The diagnostic value of motor evoked potentials. Clin Neurophysiol Off J Int Fed Clin Neurophysiol 110(7):12971307

9. Donnally CJ, Butler AJ, Rush AJ, Bondar KJ, Wang MY, Eismont FJ (2018) The most influential publications in cervical myelopathy. J Spine Surg Hong Kong 4(4):770-779

10. Harrop JS, Hanna A, Silva MT, Sharan A (2007) Neurological manifestations of cervical spondylosisan overview of signs, symptoms, and pathophysiology. Neurosurgery 60(suppl_1):S1-14-S120

11. Kato S, Oshima Y, Oka H, Chikuda H, Takeshita Y, Miyoshi K, Kawamura N, Masuda K, Kunogi J, Okazaki R, Azuma S, Hara N, Tanaka S, Takeshita K (2015) Comparison of the Japanese Orthopaedic association (JOA) score and modified JOA (mJOA) score for the assessment of cervical myelopathy: a multicenter observational study. PLoS One 10:e0123022

12. Kuijper B, Tans JTJ, Beelen A, Nollet F, de Visser M (2009) Cervical collar or physiotherapy versus wait and see policy for recent onset cervical radiculopathy: randomised trial. BMJ 339: b3883
13. Lee Y, Kim SY, Kim K (2018) A dynamic magnetic resonance imaging study of changes in severity of cervical spinal stenosis in flexion and extension. Ann Rehabil Med 42(4):584-590

14. Lyu RK, Tang LM, Chen CJ, Chen CM, Chang HS, Wu YR (2004) The use of evoked potentials for clinical correlation and surgical outcome in cervical spondylotic myelopathy with intramedullary high signal intensity on MRI. J Neurol Neurosurg Psychiatry 75(2):256-261

15. Nagoshi N, Tsuji O, Okada E, Fujita N, Yagi M, Tsuji T, Nakamura M, Matsumoto M, Watanabe K (2019) Clinical indicators of surgical outcomes after cervical single open-door laminoplasty assessed by the Japanese Orthopaedic Association Cervical Myelopathy Evaluation Questionnaire. Spinal Cord 57(8):644-651. https:// doi.org/10.1038/s41393-019-0258-4

16. Nakanishi K, Tanaka N, Kamei N, Ohta R, Fujioka Y, Hiramatsu T, Ujigo S, Ochi M (2014) Electrophysiological evidence of functional improvement in the corticospinal tract after laminoplasty in patients with cervical compressive myelopathy: clinical article. J Neurosurg Spine 21(2):210-216

17. Nouri A, Tetreault L, Zamorano J, Dalzell K, Davis A, Mikulis D, Yee A, Fehlings M (2015) Role of magnetic resonance imaging in predicting surgical outcome in patients with cervical Spondylotic myelopathy. Spine 40(3):171-178

18. Restuccia D, Di Lazzaro V, Lo Monaco M, Evoli A, Valeriani M, Tonali P (1992) Somatosensory evoked potentials in the diagnosis of cervical spondylotic myelopathy. Electromyogr Clin Neurophysiol 32(7-8):389-395

19. Restuccia D, Di Lazzaro V, Valeriani M, Aulisa L, Galli M, Tonali P, Mauguière F (1994) The role of upper limb somatosensory evoked potentials in the management of cervical spondylotic myelopathy: preliminary data. Electroencephalogr Clin Neurophysiol 92(6):502-509

20. Rikita T, Tanaka N, Nakanishi K, Kamei N, Sumiyoshi N, Kotaka S, Adachi N, Ochi M (2017) The relationship between central motor conduction time and spinal cord compression in patients with cervical spondylotic myelopathy. Spinal Cord 55(4):419-426

21. Rossini PM, Burke D, Chen R, Cohen LG, Daskalakis Z, di Iorio R, di Lazzaro V, Ferreri F, Fitzgerald PB, George MS, Hallett M, Lefaucheur JP, Langguth B, Matsumoto H, Miniussi C, Nitsche MA, Pascual-Leone A, Paulus W, Rossi S, Rothwell JC, Siebner HR, Ugawa Y, Walsh V, Ziemann U (2015) Non-invasive electrical and magnetic stimulation of the brain, spinal cord, roots and peripheral nerves: basic principles and procedures for routine clinical and research application. An updated report from an I.F.C.N. committee. Clin Neurophysiol Off J Int Fed Clin Neurophysiol 126(6): 1071-1107

22. Tamburrelli F, Di Lazzaro V, Pola E, Genitiempo M, Pilato F, Logroscino CA (2008) Cervical spondylotic myelopathy: proposal of a surveillance algorithm. Eur Rev Med Pharmacol Sci 12(3): 161-165

23. Tetreault LA, Dettori JR, Wilson JR, Singh A, Nouri A, Fehlings MG, Brodt ED, Jacobs WB (2013) Systematic review of magnetic resonance imaging characteristics that affect treatment decision making and predict clinical outcome in patients with cervical spondylotic myelopathy. Spine 38(22 Suppl 1):S89-S110

24. Tetreault L, Goldstein CL, Arnold P, Harrop J, Hilibrand A, Nouri A, Fehlings MG (2015) Degenerative cervical myelopathy: a Spectrum of related disorders affecting the aging spine. Neurosurgery 77(Suppl 4):S51-S67

25. Tykocki T, du Plessis J, Wynne-Jones G (2018) Analysis of morphometric parameters in cervical canal stenosis on neutral and dynamic magnetic resonance imaging. World Neurosurg 114:e317e322

26. Wei L, Cao P, Xu C, Wu H, Hu B, Tian Y, Yuan W (2018) Comparison of the prognostic value of different quantitative measurements of increased signal intensity on T2-weighted MRI in 
cervical Spondylotic myelopathy. World Neurosurg 118:e505e512

27. Yonenobu K, Abumi K, Nagata K, Taketomi E, Ueyama K (2001) Interobserver and Intraobserver reliability of the Japanese Orthopaedic association scoring system for evaluation of cervical compression myelopathy. Spine 26(17):1890-1894

28. Yu L, Zhang Z, Ding Q, Li Y, Liu Y, Yin G (2015) Relationship between signal changes on T2-weighted magnetic resonance images and cervical dynamics in cervical Spondylotic myelopathy. $\mathrm{J}$ Spinal Disord Tech 28:E365-E367. https://doi.org/10.1097/BSD. 0b013e31829993a8

29. Zeitoun D, El Hajj F, Sariali E, Catonné Y, Pascal-Moussellard H (2015) Evaluation of spinal cord compression and hyperintense intramedullary lesions on T2-weighted sequences in patients with cervical spondylotic myelopathy using flexion-extension MRI protocol. Spine J Off J North Am Spine Soc 15(4):668-674

30. Zhang Y-Z, Shen Y, Wang L-F, Ding W-Y, Xu J-X, He J (2010) Magnetic resonance T2 image signal intensity ratio and clinical manifestation predict prognosis after surgical intervention for cervical spondylotic myelopathy. Spine 35(10):E396-E399

Publisher's note Springer Nature remains neutral with regard to jurisdictional claims in published maps and institutional affiliations. 